\title{
Effects of hippocampal lesions on the water consumption of hooded and albino rats
}

\author{
JOHN J. BOITANO, H. GLENDON ABEL, \\ GEORGE J. HEINE, and GEOFFREY A. PATRISSI \\ Fairfield University, Fairfield, Conn. 06430
}

Simple ad lib 24-h water consumption was measured 16 days preoperatively and 60 days postoperatively in Long-Evans and Sprague-Dawley rats subjected to massive hippocampal, neocortical, or sham lesions. The only lesion effect occurred within the first 4 postoperative days, as hippocampal albinos drank more water than the control Ss. After adjusting for initial preoperative strain differences through covariance procedures, albino rats, irrespective of brain damage, consumed significantly more water than the hooded Ss for the first 16 days postoperatively. The results are contrasted with previous studies and discussed in terms of the role played by the hippocampus in mediating drinking behavior.

Current views implicate both the septum and the hippocampus in mediating response inhibition. Certain of these tasks required ablated rats to withhold approach responses to electrified water. Those that could not were said to exhibit a deficit in passive avoidance responding (PAR), provided the hypothesis of an enhanced thirst drive could be eliminated. In the case of septal lesions in rats, Lubar, Schaefer, \& Wells (1969) were able to dissociate clearly the PAR debility from the purely motivational effect of increased water intake. On the other hand, the role of the hippocampus in thirst motivation has been less unequivocal.

In two recent studies measuring simple ad lib water consumption in hippocampectomized rats, divergent results were obtained. Kimble \& Coover (1965) reported a $16 \%$ to $21 \%$ increase in water intake in hippocampal rats when contrasted with posterior neocortical or unoperated control Ss. On the other hand, Boitano, Lubar, Auer, \& Furnald (1968) did not report altered consummatory behavior for either large or small hippocampal Ss as compared with large or small neocortical or sham operates. The major difference between the two studies was the strains employed. Kimble and Coover used Sprague-Dawley albinos, while Boitano et al tested Long-Evans hooded rats. In an effort to reconcile these studies, water intake was measured in both strains before and after hippocampal, neocortical, and sham operations.

*W. Ronald Salafia sponsors this paper and takes full editorial responsibility for it.

\section{METHOD \\ Subjects}

The Ss were 23 male Long-Evans hooded rats with a mean preoperative weight of $315 \mathrm{~g}$ and 26 male Sprague-Dawley albino rats weighing $306 \mathrm{~g}$ on the average. Intrastrain preoperative matching was done on the basis of the amount of water consumed per $100 \mathrm{~g}$ of body weight. As will soon become evident, it was impossible to equate the two strains for preoperative consumption due to the heightened intake of the albinos in contrast with the hooded Ss. The matching procedure resulted in the following operative groups: (a) albino neocortical Ss $(\mathrm{N}=8)$, (b) albino shams $(\mathrm{N}=9),(\mathrm{c})$ albino hippocampal Ss $(\mathrm{N}=9)$, (d) hooded neocortical Ss $(\mathrm{N}=7)$, (e) hooded shams $(\mathrm{N}=9)$, (f) hooded hippocampal Ss $(\mathrm{N}=8)$.

\section{Surgery and Histology}

Surgery was done under sodium pentobarbital anesthesia, with a preanesthetic dose of atropine to facilitate respiration. Briefly, a midline incision exposed the cranium, which was rongeured after scraping, over the approximate A-P extent of the hippocampus. At this point, the sham-operated groups were closed by suturing. For the neocortical Ss, the neocortex overlying the corpus callosum was removed by suction, while the hippocampal animals sustained aspiration of the neocortex, corpus callosum, and hippocampus. After packing the wound with Gelfoam and closing, all animals received tetracyclene (Tetrex syrup) in their drinking water for 4 days after the operation.

At the conclusion of the experiment, the Ss were given an overdose of sodium pentobarbital and perfused intracardially with isotonic saline followed by $10 \%$ buffered Formalin. The brains were extracted, frozen, and sectioned at 40 microns. Every tenth section through the lesion area was mounted and stained.

\section{Procedure}

For 16 days preoperatively and for 60 days postoperatively, the water intake of each $\mathrm{S}$ was measured. This was done by weighing a full bottle of water on the first day, affixing it to the cage by springs, and reweighing it after $24 \mathrm{~h}$. The difference in grams was the amount consumed. This was converted directly into milliliters. With care in attaching and detaching the standard 8-oz water bottles from the cage, spillage was kept at a minimum and was estimated at less than $2 \%$ across all groups. The animals were housed in individual hanging cages, equally divided between two 30-cage racks. Ambient room temperature was $75^{\circ} \pm 4^{\circ} \mathrm{F}$. On alternate days, the body weight of each animal was determined.

\section{RESULTS \\ Histological Evaluation}

Histological examination of the brains revealed the lesion extent to be markedly similar to our previous study (Boitano et al, 1968). The only difference was the amount of posterior neocortical damage in both hippocampal groups, which was less in this study. For the hippocampal Ss, bilateral destruction was noted in the dorsal, lateral (fimbria), and posterior portions, in addition to the overlying cortex. Typically, the ventral 


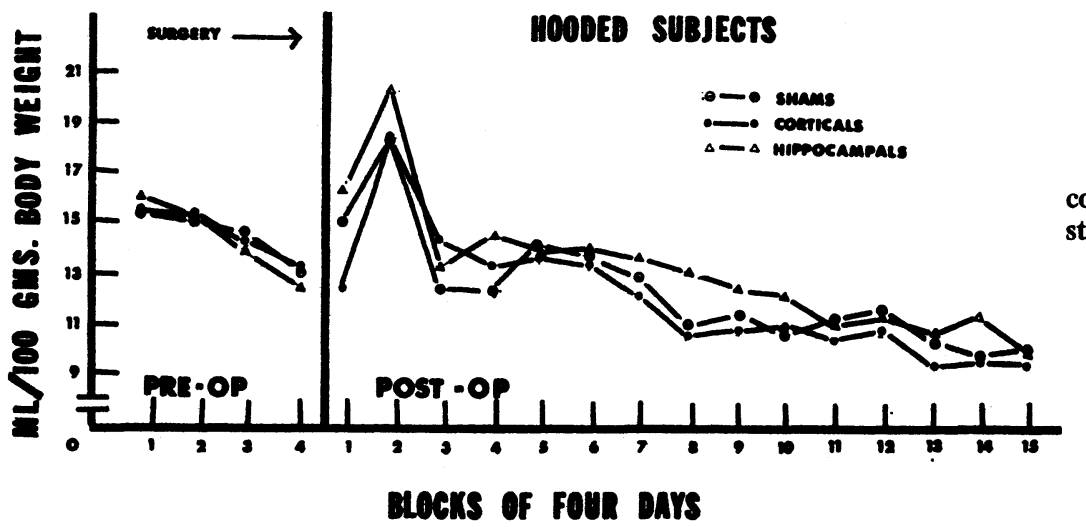

Fig. 1. Mean pre- and postoperative water consumption for the Long-Evans hooded strain.

and dorsal tips were spared, as was the posterior ventral portion. The neocortical lesions spared the cingulate gyrus and the extreme lateral entorhinal cortex. In isolated instances, the upper layer of cells in the alveus was destroyed. Thalamic gliosis, when observed, was confined to the lateral nucleus. In some animals, the lateral portion of the lateral geniculate body was also destroyed.

\section{Water Consumption}

The pre- and postoperatively ingested amounts of water for both the hooded and albino Ss are presented in Figs. 1 and 2, respectively. For both rodent strains, the data are expressed in milliliters per $100 \mathrm{~g}$ of body weight. In comparing the preoperative levels for both strains, it is evident that the albinos consumed significantly more water than the hooded Ss $(F=10.70$, $\mathrm{df}=1 / 42, \mathrm{p}<.01)$. It was this observation which precluded matching the two strains before surgery.

For the hooded Ss, there was a slight elevation in water consumption immediately after surgery (Block 1) for the shams and hippocampal animals, while the corticals actually decreased from their preoperative baseline. However, by the eighth day, all groups substantially increased their water consumption. The differences between lesioned groups for the first two blocks were statistically insignificant $(F s=1.93,1.08$, $\mathrm{df}=2 / 20$, ps $>.05)$. Following this, there was a gradual decline in water consumption extending throughout the period of observation.

After surgery, both brain-damaged albino groups exhibited marked increments in water intake when contrasted with the sham Ss $(F=9.27, \mathrm{df}=2 / 23$, $\mathrm{p}<.005)$ during the first block of 4 days. All of the intergroup comparisons proved statistically reliable (ts = 2.11 to 4.14 , ps $<.05$ to .005 ). On the eighth day, both sham and cortical Ss increased their water consumption, while the hippocampal Ss decreased. Following this, all groups exhibited a decline-first rapidly and then gradually-to the end of the observation period.

In comparing the two strains, a 2 by 4 factorial analysis of covariance with repeated measures

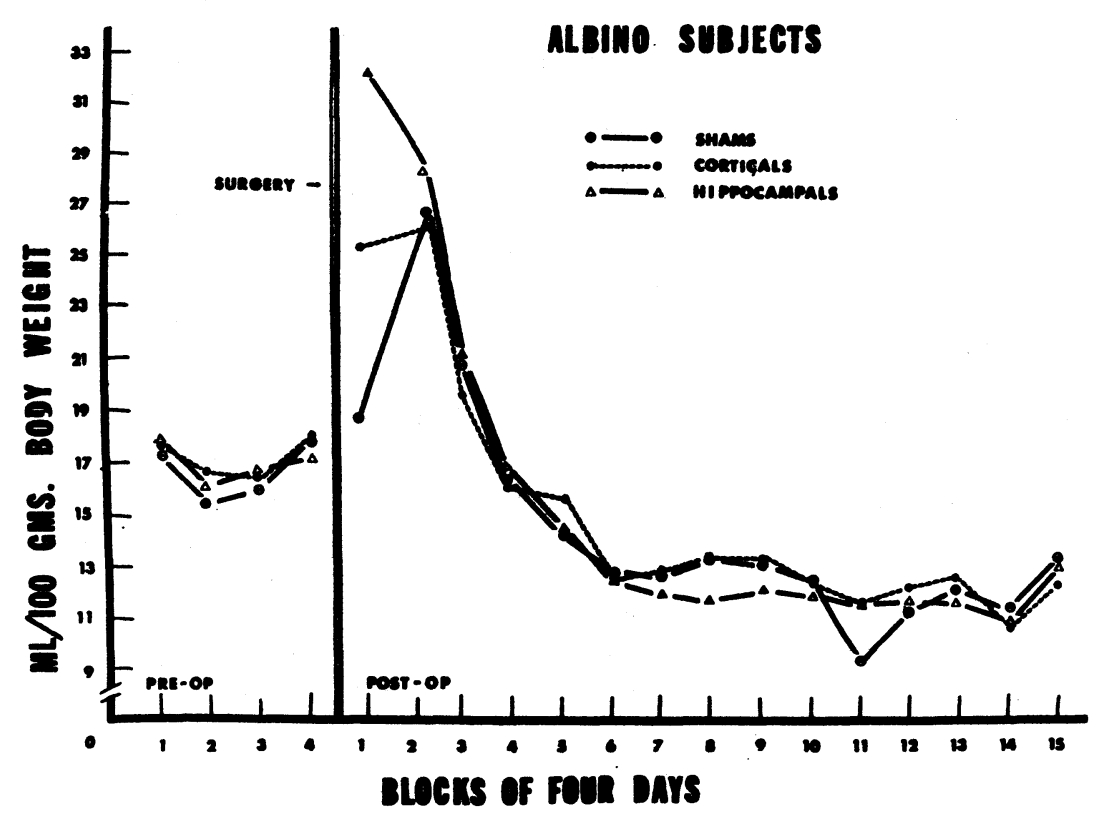

Fig. 2. Mean pre- and postoperative water consumption for the Sprague-Dawley albino strain. 
(Postoperative Blocks 1-4) was employed (Winer, 1962). Each S's preoperative 16-day mean served as the covariate. The question was whether the pronounced postoperative strain effect observed in collating Fig. 1 with Fig. 2 would be evident after adjusting the initial preoperative strain differences. Since the Ss used in the lesion groups had already been experimentally controlled by the intrastrain preoperative matching procedure, this variable was disregarded in the covariance procedure. The results of analysis of covariance revealed a highly significant postoperative strain effect after statistically equating preoperative levels $(\mathrm{F}=54.79, \mathrm{df}=1 / 45, \mathrm{p}<.001)$.

\section{DISCUSSION}

The above results suggest that albino animals, regardless of brain damage, consume more water than hooded animals during the first $\mathbf{1 6}$ days after surgery. Aside from a transient increase in water consumption for the hippocampal and neocortical albinos immediately after surgery, there is no evidence that hippocampal ablation alters water consumption when measurements are extended for 2 months postoperatively. Moreover, the pronounced increment in water consumption for the first 4 days after surgery in the albino Ss is probably due to the increased palatability of ordinary tap water adulterated by the tetracyclene antibiotic. Murphy \& Brown (1970) reported that hippocampectomized Sprague-Dawley rats consumed more of a highly palatable fluid mixture of glucose and saccharin over a 24-h period than did neocorticals or shams. On the other hand, both Murphy and Brown and Beatty \& Schwartzbaum (1968) observed an absence of sucrose preference $(8 \%)$ in hippocampal animals. In addition, both studies failed to detect significant differences in 24-h ad lib water intake measured at various short intervals after surgery.

The results of the present study, as well as our past efforts, should not be interpreted to mean that no part of the hippocampus plays a role in water consumption, or even thirst motivation. On an anatomical basis, there are extensive connections to the septal area and hypothalamus which have been involved in various aspects of drinking behavior. More recently, Holdstock (1972) has shown that rats with ventral hippocampal lesions drink significantly more water than dorsal hippocampectomized Ss or controls. While this enhanced ad lib drinking observed between Postoperative Days 30-37 may reflect the prior 17 days of a $23-$ or $47-\mathrm{h}$ deprivation schedule, it more than likely indicates dissociation of function within the hippocampus. Consequently, when lesions invade both the anterodorsal and posteroventral hippocampus, as in the present study, the result cancels any effect on simple consummatory behavior.

These interpretations still leave unexplained the Kimble and Coover findings. Our previous study equated room temperature, age of the Ss at time of surgery, and locus of the lesion. The current study additionally obviates an explanation in terms of strain differences. Although the surgery did enhance albino drinking over the first 16 days, a Lesion by Strain interaction was not evident over the entire period of observation. Until more studies are forthcoming, especially from the Oregon laboratory, the weight of increasing evidence would seem to suggest that massive hippocampal lesions do not result in an increase in simple water consumption.

\section{REFERENCES}

Beatty, W. W. \& Schwartzbaum, J. S. Commonality and specificity of behavioral dysfunctions following septal and hippocampal lesions in rats. Journal of Comparative \& Physiological Psychology, 1968, 66, 60-68.

Boitano, J. J., Lubar, J. F., Auer, J., \& Furnald, M. Effects of hippocampectomy on consummatory behavior and movement-inhibition in rats. Physiology \& Behavior, 1968, 3, 901-906.

Holdstock, T. L. Dissociation of function within the hippocampus. Physiology \& Behavior, 1972, 8, 659-667.

Kimble, D. P. \& Coover, G. D. Effects of hippocampal lesions on food and water consumption in rats. Psychonomic Science, 1966, 4, 91-92.

Lubar, J. F., Schaefer, C. F., \& Wells, D. G. The role of the septal area in the regulation of water intake and associated motivational behavior. Annals of the New York Academy of Sciences, 1969, 157, 875-893.

Murphy, H. M. \& Brown, T. S. Effects of hippocampal lesions on simple and preferential consummatory behavior in the rat. Journal of Comparative \& Physiological Psychology, 1970, 72, 404-415.

Winer, B. J. Statistical principles in experimental design. New York: McGraw-Hill, 1962. Pp. 606-615.

(Received for publication October 22, 1972.) 